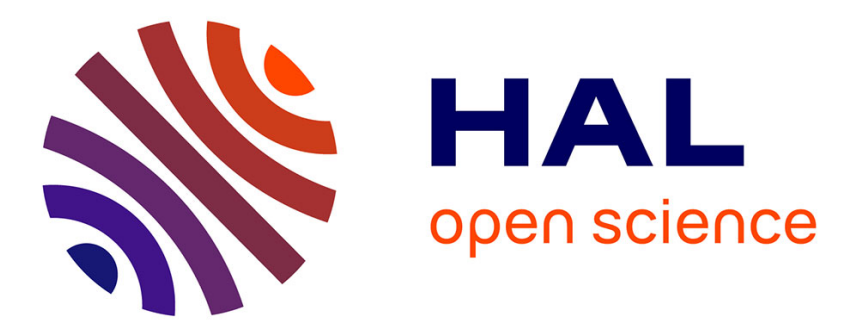

\title{
2.5@C - An Off-Axis Telescope Concept for Antarctic Astronomy
}

G. Moretto, Nicolas Epchtein, Maud Langlois, Isabelle Vauglin

\section{To cite this version:}

G. Moretto, Nicolas Epchtein, Maud Langlois, Isabelle Vauglin. 2.5@C - An Off-Axis Telescope Concept for Antarctic Astronomy. Proceedings of the Society of Photo-Optical Instrumentation Engineers, 2012, 8444, pp.84445E. 10.1117/12.927257 . in2p3-00740937

\section{HAL Id: in2p3-00740937 https://hal.in2p3.fr/in2p3-00740937}

Submitted on 11 Oct 2012

HAL is a multi-disciplinary open access archive for the deposit and dissemination of scientific research documents, whether they are published or not. The documents may come from teaching and research institutions in France or abroad, or from public or private research centers.
L'archive ouverte pluridisciplinaire HAL, est destinée au dépôt et à la diffusion de documents scientifiques de niveau recherche, publiés ou non, émanant des établissements d'enseignement et de recherche français ou étrangers, des laboratoires publics ou privés. 


\title{
An Off-Axis Telescope Concept for Antarctic Astronomy
}

\author{
Gil Moretto $^{*(1)}$, Nicolas Epchtein ${ }^{(2)}$ Maud Langlois $^{(3)}$ and Isabelle Vauglin $^{(3)}$ \\ (1) Lyon Institute of Origins, Institute of Nuclear Physics of Lyon, IN2P3-CNRS, \\ Université de Lyon, F-69622, Lyon, France \\ (2) Laboratory J-L. Lagrange, INSU-CNRS, Nice, France \\ (3) Center for Astrophysics Research of Lyon, INSU-CNRS, Saint-Genis Laval, France \\ Université de Lyon, F-69622, Lyon, France
}

\begin{abstract}
The Antarctic plateau offers exceptional atmospheric and environmental conditions for astronomical observations over a wide range of wavelengths and uniquely favorable to infrared astronomy. Exceptional low sky brightness throughout the near- and mid-infrared and a telescope facility complying with the highest possible dynamic range for photometry, angular resolution and the wide-field leads to the possibility of a modest-sized $2 \mathrm{~m}$ off-axis telescope achieving comparable sensitivity to that of a larger ground-based $8-10 \mathrm{~m}$ class telescope or a same sized space-based ones.
\end{abstract}

Keywords: infrared cases for Antarctica, off-axis telescope, low scattered light, low emissivity.

\section{INTRODUCTION}

We address here the scientific relevancy and feasibility of creating an astronomical observatory for the optical/infrared spectral range on the Antarctic Plateau/Dome C. The properties of the atmosphere above the Antarctic Plateau are known to be unique on the Earth surface. Atmospheric turbulence is concentrated in a thin layer of a few tens of meters, the sky opacity, particularly in the infrared, is considerably reduced and the thermal infrared sky background radiation is lower by a factor of 10 to 20 in the $2-3 \mu \mathrm{m}$ windows. The combination of these advantages makes a $2 \mathrm{~m}$ class telescope comparable to an $8 \mathrm{~m}$ one in a conventional site in terms of sensitivity. The Antarctic Plateau is thus likely to become in the coming decades a new major platform for advanced astronomical observations. In the short/medium term, it is expected that synoptic infrared imaging and spectro-imaging surveys exploring the temporal dimension, from Antarctica could play an essential role in the context of the future large ground based and space projects such as E-ELT, JWST, LSST, EUCLID, GAIA. A medium/large aperture telescope on the Antarctic Plateau have the potential to undertake tasks previously thought to be possible only in space, for example the imaging and crude spectroscopy of Earth-size extra-solar planets (Angel, Lawrence and Storey, 2004).

Three astrophysical key-domains for an optical/IR facility had been identified, for instance in the ARENA vision (2010), namely (1) the search and characterization of Type Ia supernovae and the investigation of the distant Universe, (2) the stellar populations and evolution in the local group of galaxies, (3) the identification and characterization of extra-solar planets. We present here a preliminary new concept of telescope able to achieve the three above mentioned key-domains. Such concept offers a wide range of cutting edge technological investigations that would definitely overcome the scope of Antarctic astronomy.

* G. Moretto: E-mail: Gil.Moretto@ipnl.in2p3.fr, Telephone: 33 472.431.108

Ground-based and Airborne Telescopes IV, edited by Larry Stepp, Roberto Gilmozzi, Helen J. Hall, Proc. of SPIE Vol. 8444, 84445E • (C) 2012 SPIE • CCC code: 0277-786/12/\$18 • doi: 10.1117/12.927257 


\section{DOME C SITE QUALITY}

The Dome $\mathrm{C}$ unique quality has been reported for day- and night-time astronomical observations and presented by Aristidi et al. (2009) and Gredel (2009). At a glance here below are the main results of atmospheric measurements carried out, so far:

Precipitable water vapour (PWV) - Monthly average ranges from $0.72(+/-0.20) \mathrm{mm}$ in December to $0.26(+/-0.1) \mathrm{mm}$ in March/April period. Observations in the $200 \mu \mathrm{m}$ window opens at a transmission level of about $20 \%$ during $25 \%$ of the time. Observations at $350 \mu \mathrm{m}$ and $450 \mu \mathrm{m}$ are possible all year round (see, Tremblin et al., 2011). Note that low PWV also results in an improved transmission in the NIR and MIR windows and in an extended wavelength coverage (notably in the important $3 \mu \mathrm{m}$ window).

Sky Brightness - Beyond $2.3 \mu \mathrm{m}$, the dominant factor is the emission of the molecules (mainly $\mathrm{H}_{2} \mathrm{O}$ ) and aerosols at the temperature of the atmosphere $\left(-50^{\circ} \mathrm{C}\right.$ to $\left.-90^{\circ} \mathrm{C}\right)$. Thanks to these lower temperatures and the lower amount of PWV, the sky is dramatically darker than in the other observatories by at least 3 magnitudes (Phillips et al., 1999; Walden et al., 2005). In the visible, sky is dark at solar elevation of $-12^{\circ}$ resulting in a total of 2506 dark hours per year. The fraction of clear nights is of the order of $80-85 \%$ during winter.

Temperature and Wind Profiles - Quasi-periodic ground temperature oscillations occur during summer and winter months. In winter $\mathrm{dT} / \mathrm{dt}$ is up to $30^{\circ} \mathrm{C} /$ week., in summer $\mathrm{dT} / \mathrm{dt}>10^{\circ} \mathrm{C} / \mathrm{hour}$. Wind speed is very low (Aristidi et al., 2005). Ground layer wind profiles do not show any strong diurnal variations.

Seeing - A summary of the seeing measurements at Dome $\mathrm{C}$ has been reported by Aristidi et al. (2009) and Ziad et al. (2008). Data obtained with three DIMMs set up at $3 \mathrm{~m}, 8 \mathrm{~m}$, and 20m height above the ground level show that the seeing is of poor quality on the ground because of a turbulent boundary layer of mean height $\sim 23 \mathrm{~m}$ in winter. However, the free atmosphere seeing above the boundary layer is indeed very good with a median value of $\sim 0.36 \operatorname{arcsec}$ (Aristidi et al., 2009). The turbulent layer below the boundary layer can be corrected very efficiently using adaptive optics allowing to achieve 0.40 arcsec over 60 degrees FOV in $\mathrm{H}$ and $\mathrm{K}$ bands (Le Roux et al., 2007).

\section{AN OFF-AXIS TELESCOPE FOR DOME C}

Astrophysical key programs as identified above (1)+(2)+(3) for an optical/IR facility call for three driver cases (a) the highest possible dynamic range for photometry, (b) angular resolution and (c) the wide-field imaging in the optical and the thermal infrared. A telescope facility complying with the three driver cases $(\mathbf{a})+(\mathbf{b})+(\mathbf{c})$ and capitalizing the Dome $\mathrm{C}$ outstanding atmospheric and environmental performance over the optical and thermal infrared wavelengths, assert for an off-axis telescope concept.

Diffraction limited images of faint objects should be soon obtained with existing 8-m class ground based telescopes, equipped with laser guide stars adaptive optics systems. Ultra deep images, particularly in the $2-5 \mu \mathrm{m}$ region where the natural zodiacal background is very low, should be obtained by the James Webb space telescope, operating at cryogenic temperature. Observations to complement the JWST will be possible from the ground, they will require a very large telescope with the lowest possible thermal background and excellent emissivity and scattered light performance. 


\section{AstroConcordia, January 2011}

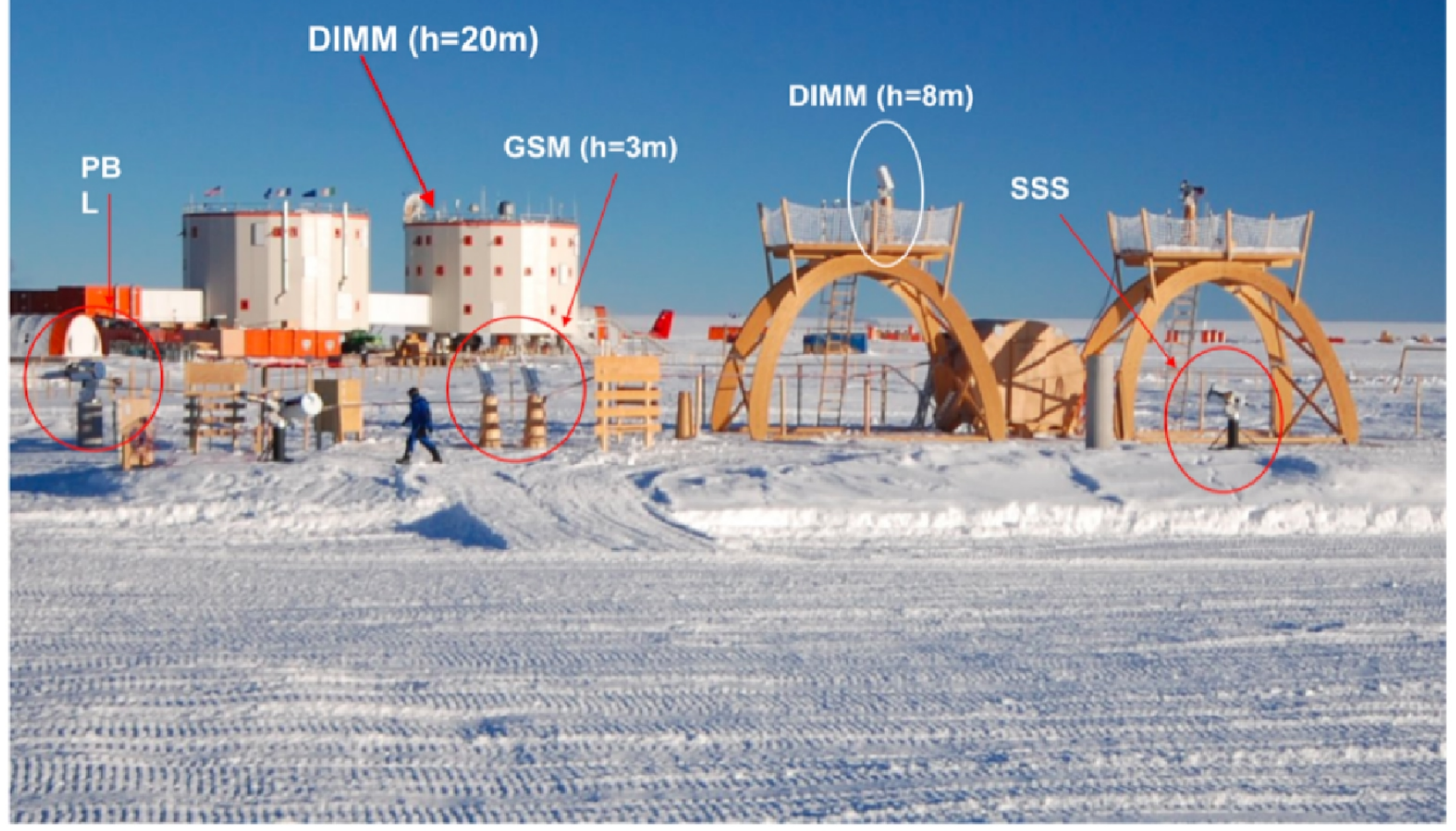

(A)

WINTER $\mathrm{h}=23 \mathrm{~m} \quad$ Seeing $=0.36$ Arcsec

\begin{tabular}{|c|c|c|c|}
\hline & Seeing & Isop. & Coh. time \\
\hline DIMM/GSM & 0.4 "' & $4.1^{\prime \prime}$ & \\
\hline SSS & $0.3^{\prime \prime}$ & $6.9^{\prime \prime}$ & $10.2 \mathrm{~ms}$ \\
\hline Balloons & $0.4 "$ & $2.7 "$ & $6.8 \mathrm{~ms}$ \\
\hline AASTINO $2004^{1}$ & $0.3^{\prime \prime}$ & $5.7^{\prime \prime}$ & $7.9 \mathrm{~ms}$ \\
\hline Simulations $^{2}$ & $0.3 "$ & & \\
\hline
\end{tabular}

\begin{tabular}{|l|l|l|l|}
\hline Mauna Kea & $0.6^{\prime \prime}$ & $1.9^{\prime \prime}$ & $2.7 \mathrm{~ms}$ \\
\hline Paranal & $0.8^{\prime \prime}$ & $2.6^{\prime \prime}$ & $3.3 \mathrm{~ms}$ \\
\hline
\end{tabular}

(B) 'Lawrence et al 2004
'Lascaux et al 2011
SUMMER $\mathrm{h}=8 \mathrm{~m}$ Seeing $=0.4$ Arcsec

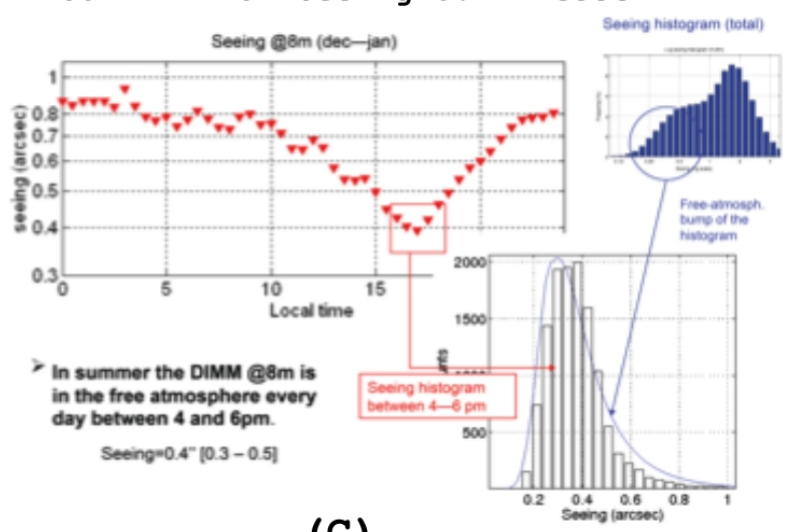

(C)

Figure 1 - (A) The French/Italian Astro Concordia base at Dome C - showing in the backline the staff headquarters (two cylindrical structures) and in the frontline the seeing measurements instruments. The data for winter and summer seeing measurements are presented respectively by (B) and (C), from Aristidi et al. (2009). 
An off-axis telescope provides an inherently low scattered light design because there are no obstructions in the beam. There are a minimal number of scattered light sources. All mirrors can be robustly supported and articulated because of the easy access allowed by this design. All warm components, sources of the telescope self-thermal emission, will be out of optical beam minimizing its emissivity.

Here we refer to an off-axis telescope configuration as an off-axis, or decentered section of a concentric, on-axis optical configuration in which the primary and auxiliary mirrors are an off-axis piece of a concentric parent mirror. Optically speaking an off-axis telescope is not an asymmetric system, it is a decentered system preserving its bi-lateral symmetry. This means that the system preserves many of the optical performances, tolerances and sensitivities characteristics of the parent concentric system - a deal for system opto-mechanical alignment. In fact, since only a small section of the parent optical system is illuminated, some geometrical performance characteristics of the daughter system supersede the parent optics.

A three-mirror decentered Paul-Baker (Paul, 1935; Baker, 1969) system is our guideline concept reference, where some variation on the shape of the three mirrors is allowed to achieve good image quality across a wide field of view. One of the main constraints during such optimizations is the necessity to have compact designs, making it possible to be installed on top of a $23 \mathrm{~m}$ high (or higher) tower to overcome the boundary layer developed some $2 \mathrm{~m}$ above ice and up to $23 \mathrm{~m}$ (Gredel 2009). Above that, the free atmospheric seeing reach its best value during the winter, see Figure 1 (Aristidi et al., 2009).
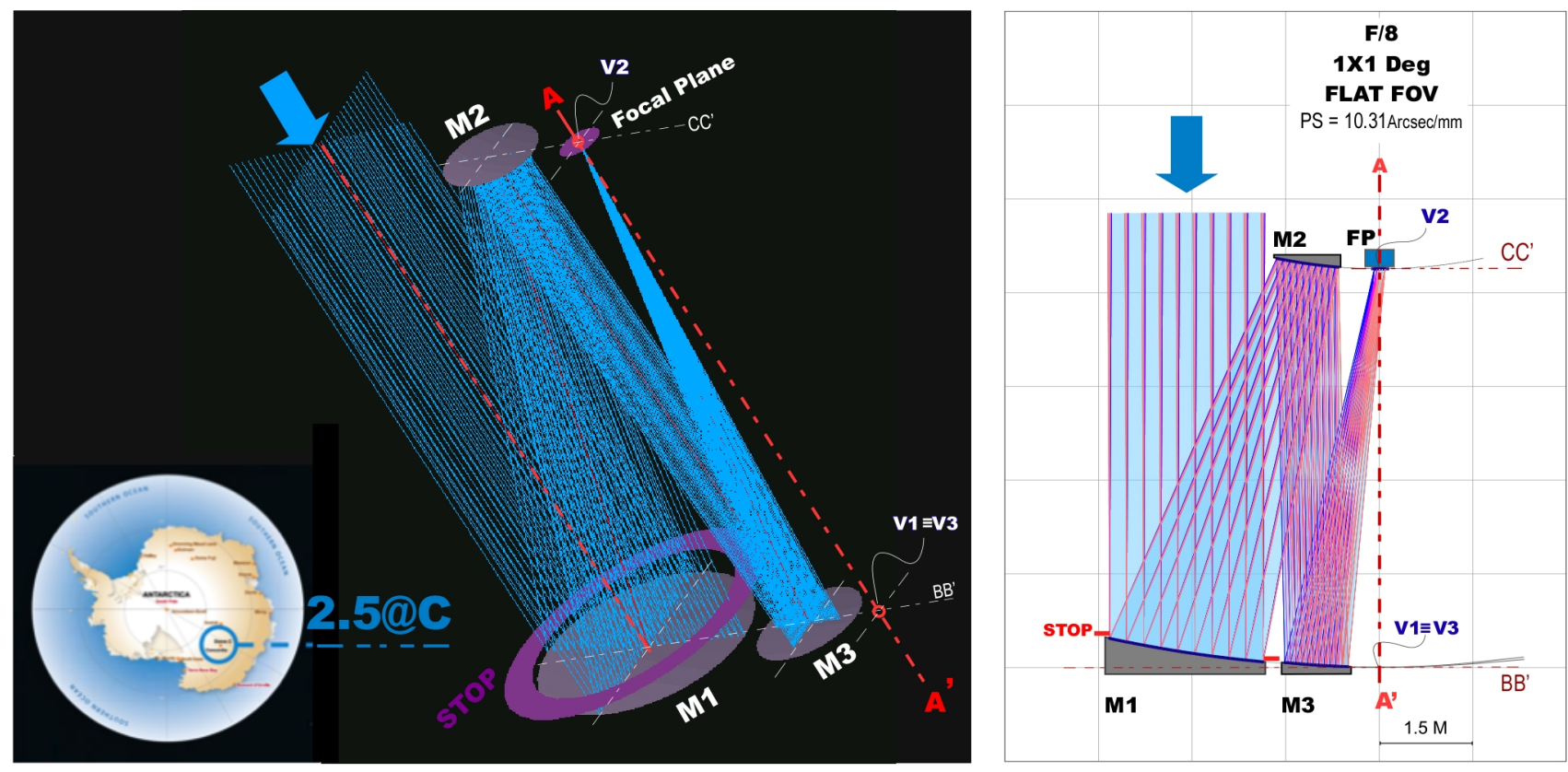

Figure 2 - The 2.5@C an off-axis telescope for Dome C. A 2.5 meter unobstructed aperture M1 yielding in a F/8 system optimized over a 1x1 Deg FOV. AA' is the parent mirrors M1, M2 and M3 optical axis. M1 and M3 vertexes V1 and V3 are coincident as well as for M2 (V2) and FP. Note that the primary mirror M1, the secondary M2 and the tertiary M3 are not tilted mirrors but are a decentered piece of concentric system, preserving its bi-lateral symmetry. 
A baseline design for Dome $\mathrm{C}$ relies on a 2.5 meter unobstructed aperture $\mathrm{M} 1$ which should produce a F/8 system optimized over an 1x1 Deg FOV, hereafter dubbed the 2.5@C. We generate a PaulBaker configuration by adding auxiliary convex secondary mirror (M2) and a concave tertiary (M3). This configuration produces excellent correction across wide FOV's but typically (concentric configuration) also suffers from a lot of obscuration because the large size of the secondary and the tertiary mirrors. Also a concentric configuration produces a trapped focal plane between M2 and M3. This obscuration is avoided if we consider a decentered (off-axis) optical configuration.

The decision on plate scale $(\mathrm{PS}=10.31 \mathrm{Arcsec} / \mathrm{mm})$ was a compromise between an optimal sampling for high resolution and a compact design. An effective focal length (EFL) of $20 \mathrm{~m}$ produces a support structure long of V1V2 $=\mathrm{V} 1 \mathrm{FP}<7 \mathrm{~m}$, short enough for our proposes. As shown in Figure 3 we consider a typical detector pixel size of $15 \mu \mathrm{m}$, which for an $\mathrm{EFL}=20 \mathrm{~m}$ results in a sampling of FWHM=2.35 pixels. This is the Nyquist criterion, the standard deviation. Some discussion has been flown confirming that a sampling of FWHM=3.5 pixels or more is the optimal for high resolution.

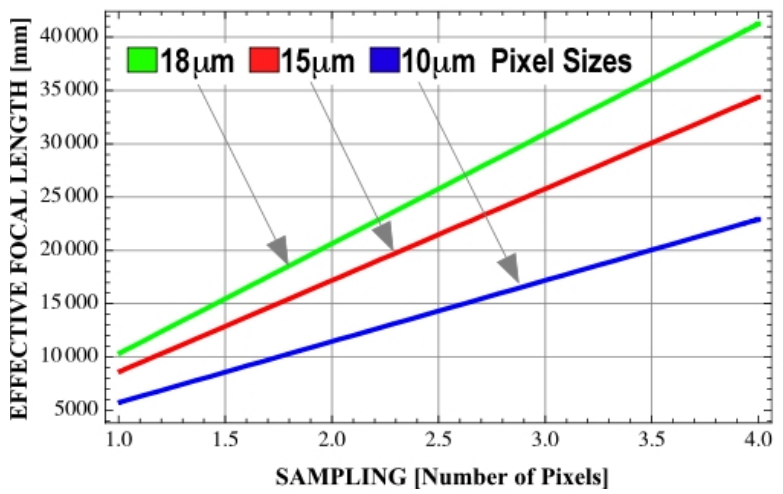

Figure 3 - The EFL versus sampling for several commercially ready pixel sizes and considering a seeing of 0.36 Arcsec for the Dome C.

In such case we better consider pixel size of $10 \mu \mathrm{m}$ for our design as shown in Figure 3 . Such pixel size should be technology off-shelves by the end of 2012!

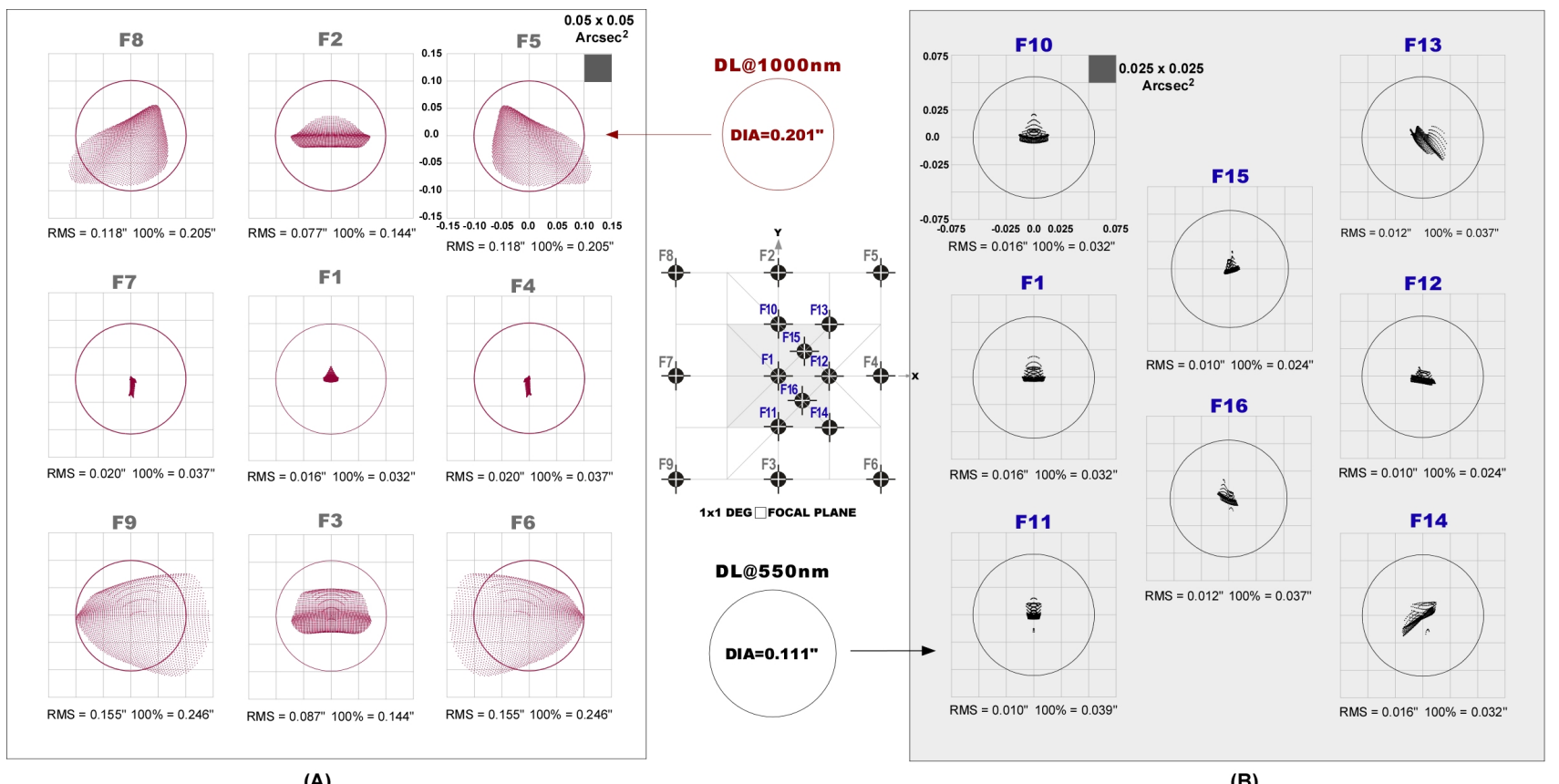

(A)

(B)

Figure 4 - The 2.5@C geometric optical performance over a flat FOV. (A) The PSF computed on the edge and center of a 1X1 Deg ${ }^{2}$ FOV. The PSF computed across a $1 / 2 \times 1 / 2 \mathrm{Deg}^{2} \mathrm{FOV}$ is shown in (B). DL stands for diffraction limit diameter at $1000 \mathrm{~nm}$ and $550 \mathrm{~nm}$ wavelength. Note in (A) the spots F8\&F5, F7\&F4 and F9\&F6 confirm the optical bi-lateral symmetry of a decentered system. 
The optical performance at the edges of $1 \times 1 \mathrm{Deg}^{2} \mathrm{FOV}$ is show in Figure 4. The PSF spots at corneredges (F5, F6, F8, F9) of $1 \mathrm{X} 1 \mathrm{Deg}^{2}$ FOV are diffraction limited at $1000 \mathrm{~nm}$ wavelength and elsewhere they are diffraction limited at $550 \mathrm{~nm}$ or even better. Note that the blur in this system is only weakly dependent on the off-axis angle and the telescope will be entirely seeing limited.

The optical performance and opto-mechanical characteristic of such concept show its versatility and optimal solution to minimize the light scattering and emissivity and capitalizing the Antarctica/Dome $\mathrm{C}$ exceptional seeing and sky transparence on near and thermal-IR.

In terms of feasibility there are several myths about off-axis telescopes. The assessment of new technologies count on the off-axis telescopes already built, for example SOLARC ${ }^{\mathrm{A}}$ and $\mathrm{NST}^{\mathrm{B}}$. Both act as pathfinders for future off-axis telescope projects to improve the maturity of evolving technologies; enabling more thoroughly understand how to overcome the technical and engineering challenges specific to this kind of design. In particular, resolving issues of alignment, heat dissipation and guiding off of asymmetrical phenomena.

A preliminary opto-mechanical sensitivity for the $6.5 \mathrm{~m}$ off-axis $\mathrm{NPT}^{\mathrm{C}}$ was done by Moretto and Kuhn (2000). The NPT opto-mechanical system required accurate positioning to $0.1 \mathrm{~mm}$ and $20-40$ arcsec. Even a relatively floppy mechanical mount with a flexure of $1 \mathrm{~mm}$ in combination with commercial hexapod secondary mount mechanisms and a simple metrology system should be able to maintain telescope collimation. As the 2.5@C the NPT mirrors (M1, M2, and M3) do not physically contain their vertices (so there is no intersection between them and the parent primary mirror optical axis), it is straightforward to define an optical and mechanical fiducial surface near the focal plane that is referenced to the off-axis primary mirror for deterministic or active metrology. In view of the 2.5@C's M1, M2, M3 and FP vertices co-linearity (it was not the case for the NPT), it should make easier its opto-mechanical sensitivities and its alignment and collimation.

The possibility of building a large off-axis optical telescope depends critically on the feasibility of the optics (see Moretto, Kuhn and Goode, 2012, at this conference). Steward Observatory Mirror Lab (SOML) (Arizona, USA) had just finish to polish the largest off-axis segment mirror so far; an $8.4 \mathrm{~m}$ in diameter off-axis paraboloid mirror. The most critical parts of polishing and tests have been demonstrated in the polishing and measurement of a $1.7 \mathrm{~m}$ off-axis prototype and the primary mirror for the New Solar Telescope (NST) (see Martin et al., 2012, at this conference). Sagem (France), through its Reosc department has also started polishing and testing prototypes segments for the ELTM1 (see Rodolfo et al., 2012, at this conference)

\footnotetext{
A SOLAR C - Scatter-free Observatory for Limb Active Regions and Coronae is a $0.5 \mathrm{~m}$ off-axis coronagraphic reflecting telescope located on Haleakala adjacent to the Mees Solar Observatory. Institute for Astronomy, University of Hawaii.

B NST - New Solar Telescope is a $1.6 \mathrm{~m}$ off-axis New Solar Telescope at the BBSO/NJIT. A collaboration with the University of Hawaii and the Korea Astronomy \& Space Science Institute. See Goode et al (2012) at this conference.

${ }^{C}$ NPT - New Planetary Telescope, a $6.5 \mathrm{~m}$ off-axis telescope design study, encompassing many of the mechanical, optical fabrication issues, and facility concerns, has been undertaken by a NPT study group that was organized by the Institute for Astronomy and the Infrared Telescope Facility (IRTF) during 1999. To support the NASA mission, the primary objectives of the NPT are studies of Kuiper Belt objects, near-Earth objects, and direct support of space missions such as Cassini.
} 


\section{CONCLUSIONS}

Many areas of modern astrophysics are not flux-limited but are rather dynamic-range limited. Simply collecting more photons will not solve the problem - for these topics, we don't need bigger telescopes, we need better telescopes set up in exceptional sites.

A better telescope concept, based on an off-axis optical design that allows exceptional dynamic range for photometry and high angular resolution + wide field imaging and capitalizes the exceptional atmospheric and environmental Antarctic conditions for astronomical observations over the optical and thermal infrared wavelengths is proposed.

The Antarctic Plateau and, in particular the French-Italian station Concordia, is indeed likely to provide in the future the best site on Earth to set up a medium size infrared telescope facility thank to both, exceptional turbulence at low height and minimal sky brightness conditions. To be costefficient and to take maximum benefit from this unique environment, the instrument and focal instrumentation must be fully optimized. For this reason, we propose an off-axis telescope concept that minimizes stray light due to the diffraction by mirror supports, and instrumental thermal selfemissivity. Since most of the turbulence is concentrated in a thin layer, the telescope must be set up above the boundary layer, i.e., at about $30 \mathrm{~m}$ above the ground at Dome $\mathrm{C}$ (perhaps at lower elevation in even better sites) and makes use of Adaptive optics device to alleviate the residual turbulence.

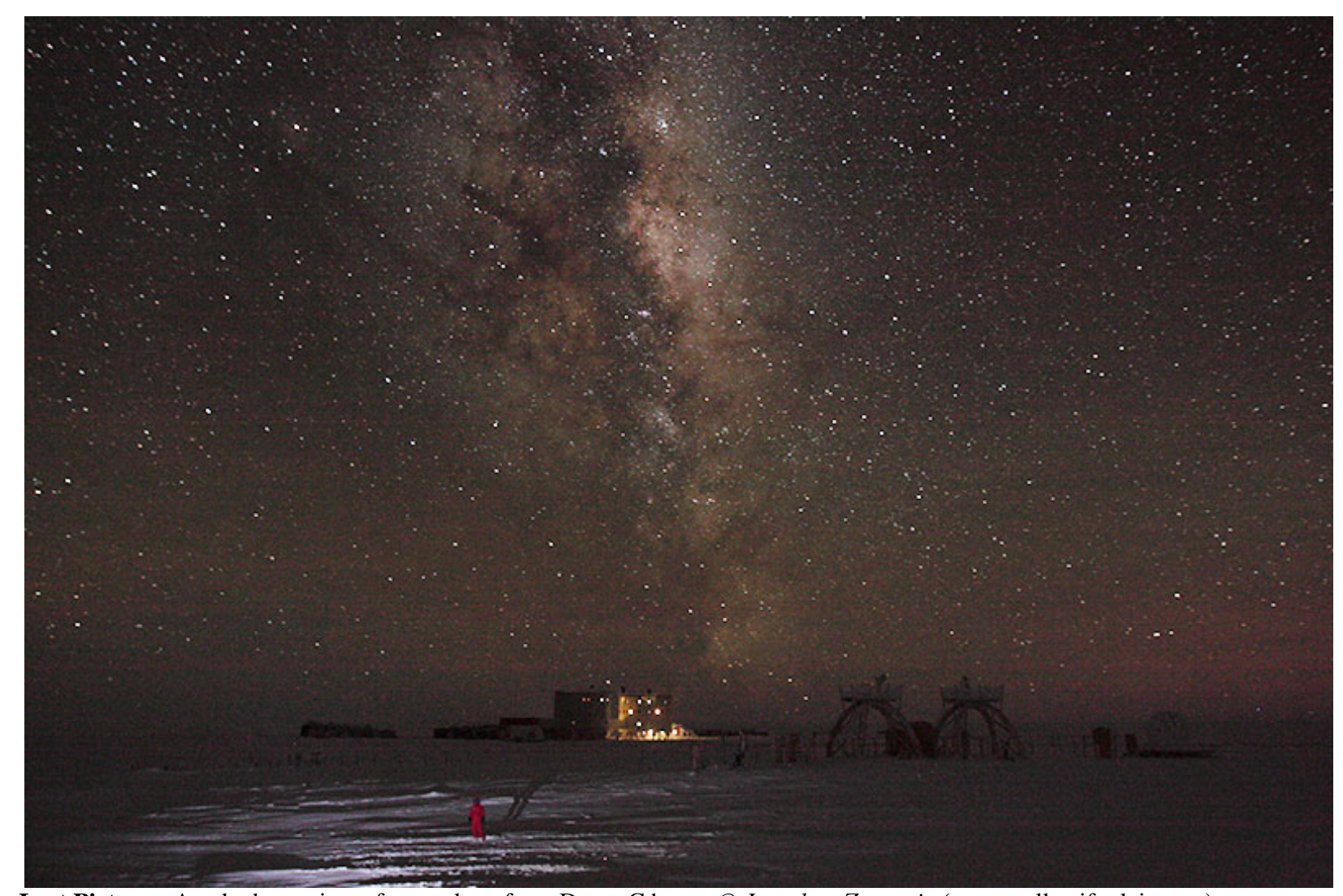

Last Picture - A naked eye view of our galaxy from Dome C base - (C) Jonathan Zaccaria (www.collectifpolaire.org). 


\section{REFERENCES}

1. Angel, R., Lawrence, J., Storey J., Concept for a second Giant Magellan Telescope (GMT) in Antarctica,. Pros. SPIE 5382 (Part 1), 76-84, 2004.

2. ARENA consortium, $A$ vision for European Astronomy and Astrophysics at the Antarctic station Concordia, Dome C in the next decade 2010-2020, ed., N. Epchtein, Publisher Nova Terra.

3. Aristidi, E., et al., An analysis of temperatures and wind speeds above Dome, Antarctica, A\&A 430, 739, 2005.

4. Aristidi, E. et al., Dome C site testing: surface layer, free atmosphere seeing, and isoplanatic angle statistics, A\&A 499, 955, 2009.

5. Baker J.G., On Improving the Effectiveness of Large Telescopes, IEEE Transactions of Aerospace and Electronic System, AES-5, 261, 1969.

6. Goode P. R. and Cao W., The 1.6m new solar telescope (NST) in Big Bear, paper 8440-01 at this conference, 2012.

7. Gredel, R., Site characterization at Dome C-The ARENA work, Proc. $3^{\text {rd }}$ ARENA Conf., eds. L. Spinoglio \& N. Epchtein, EDP EAS Ser. Vol. 40, 11, 2010

8. Kuhn, J.R., Howley S.L., Some Astronomical Performance Advantages of Off-Axis Telescopes, PASP, 111, 1999.

9. Kuhn, J.R., Moretto, G., Racine F., Roddier F., Coulter, R., A Large Aperture, High Dynamic Range Telescope Concept, PASP, 113, 2001.

10. Le Roux, B., Langlois, M., Carbillet, M., Fusco, T., Ferrari, M., Burgarella D., Ground layer adaptive optics for dome c: Optimisation and performance, OSA Adaptive Optics: Analysis and Method (2007)

11. Martin H. M., Allen R. G., Burge J.H., et al., Production of $8.4 m$ segments for the Giant Magellan Telescope, paper 8450-90 at this conference, 2012.

12. Moretto, G., Kuhn, J.R. and Goode Ph., Reviewing off-axis telescope concepts: a quest for highest possible dynamic range for photometry and angular resolution, paper 8444107 at this conference, 2012.

13. Moretto, G. and Kuhn, J.R. , Off-Axis System for 4m Class Telescope, Applied Optics, Vol. 37 , No 16, 1998.

14. Moretto, G. and Kuhn, J.R., Optical Performance of the 6.5-m Off-Axis New Planetary Telescope, Applied Optics, Vol 39, N0. 16, 2000

15. Paul M., Systèmes Connecteurs pour Réflecteurs Astronomique, Rev. Opt. 14, 169, 1935.

16. Phillips A., et al., The near-infrared sky emission at the South Pole in Winter, ApJ, 527, 1009, 1999.

17. Rodolfo, J. F. et al., Prototypes segment polishing and testing for the ELT M1, paper 8450-84 at this conference, 2012.

18. Tremblin, P. et al., Site testing for submillimetre astronomy at Dome C, Antarctica, A\&A 535, $112,2011$.

19. Walden, V.P., et al., First Measurements of the infrared sky brightness at Dome C Antarctica, PASP, 117, 300, 2005.

20. Ziad, A. et al., First statistics of the turbulence outer scale at Dome C, A\&A 491, 917, 2008 